\title{
Efficacy and Prognostic Analysis of 315 Stage I-IVa Esophageal Cancer Patients Treated with Simultaneous Integrated Boost-Intensity-Modulated Radiation Therapy
}

\section{Peng Cai (iD \\ Yan Yang \\ Duo-jie Li}

Department of Radiotherapy, The First Affiliated Hospital of Bengbu Medical College, Bengbu, 233004, People's Republic of China
Correspondence: Duo-Jie Li

Department of Radiotherapy, The First Affiliated Hospital of Bengbu Medical

College, No. 287 of Changhuai Road,

Bengbu, 233004, People's Republic of China

Tel +8613956332626

Email liduojieI23@163.com
Purpose: Use of simulated integrated boost-intensity-modulated radiation therapy (SIBIMRT) is rarely reported in the treatment of esophageal cancer. This study was performed to observe the curative effect and prognostic factors associated with concurrent chemoradiotherapy for esophageal cancer using modern radiotherapy (RT) techniques.

Patients and Methods: In total, 315 patients with esophageal squamous cell carcinoma who received SIB-IMRT between 2015 and 2018 were included in this retrospective study. Median doses were planning target volume (PTV) $5400 \mathrm{cGy}, 30$ times (180cGy/fraction); planning gross tumor volume (PGTV) $6000 \mathrm{cGy}, 30$ times (200 cGy/fraction), once a day and 5 times a week. The entire period of RT was 6 weeks. Overall survival (OS), progression-free survival (PFS), objective response rate (ORR), and adverse reactions were observed. Univariate analysis was performed, and factors with $\mathrm{P}<0.15$ were included in multivariate analysis. Cox regression analysis was used for multivariate prognostic analysis. $\mathrm{P}<0.05$ was considered statistically significant. The incidence of adverse reactions under single chemotherapy concurrent chemoradiotherapy (sCCRT) and double chemotherapy concurrent chemoradiotherapy (dCCRT) was analyzed.

Results: Two-year, 3-year OS and PFS of the entire group were $49.5 \%, 40.2 \%$ and $40.3 \%$, $34.0 \%$, and the median survival time was 23.5 months. Univariate and multivariate analyses showed that $\mathrm{T}$-stage $(\mathrm{P}=0.049), \mathrm{N}$-stage $(\mathrm{P}=0.024)$, clinical stage $(\mathrm{P}=0.041)$, short-term efficacy $(\mathrm{P}<0.001)$, and use of concurrent chemotherapy $(\mathrm{P}<0.001)$ were the influencing factors for OS. ORR was $87.6 \%$. Adverse reactions were significantly increased with increasing chemotherapy dose.

Conclusion: The adverse reactions of SIB-IMRT in esophageal cancer can be tolerated. T-stage, N-stage, clinical stage, short-term curative effect, and concurrent chemotherapy are the prognostic factors affecting survival. Because it has lower toxicity and is as effective as dCCRT, sCCRT should be considered in the management of esophageal cancer.

Keywords: esophageal neoplasm, prognosis, intensity-modulated radiotherapy, SIB-IMRT

\section{Introduction}

Esophageal cancer is the seventh most common cancer globally and has a poor prognosis. The incidence of esophageal cancer is highest in East Asia, with China being the leading region. ${ }^{1}$ Surgery is the recommended treatment for early esophageal cancer. ${ }^{2}$ However, approximately two-thirds of esophageal cancer patients are in the local advanced stages of treatment, which prevents radical surgical resection. 
A prospective randomized controlled study showed that radical concurrent chemoradiotherapy (CCRT) was as effective as surgery. Rates of progression-free survival (PFS) and overall survival (OS) following radiotherapy (RT) are similar to those achieved with standard resection for potentially resectable esophageal squamous cell carcinoma. $^{3}$

Following rapid developments in RT technology, the intensity-modulated radiation therapy (IMRT) technique is able to increase the dose to the tumor target after irradiating the tumor target volume and the clinical target volume range. Simulated integrated boost (SIB)-IMRT technology can directly increase the tumor target area, and deliver different segmented doses to different target areas. Compared with IMRT techniques, the single-dose and total dose delivered with SIB-IMRT is increased, with greater destruction of tumor tissue. Dosimetry studies have shown that with the application of SIB RT, one can successfully increase the dose to boost areas without increasing adverse reactions. ${ }^{4,5}$ The long-term curative effect of SIB-IMRT in the treatment of esophageal squamous cell carcinoma is rarely reported, while sample sizes of previous studies have been small. The present study aims to investigate the clinical efficacy and related prognostic factors of SIB-IMRT in esophageal squamous cell carcinoma.

\section{Materials and Methods Inclusion and Exclusion Criteria}

In our retrospective study, 315 patients who were either unresectable or refused surgery between January 2015 and December 2018 were analyzed at the First Affiliated Hospital of Bengbu Medical College. Patients fulfilling the following criteria were included: histologically proven esophageal squamous cell carcinoma; eligible for SIBIMRT for specific RT; previously untreated; Karnofsky performance status score $\geq 90$; white blood cell count $\geq 4 \times 10^{9} / \mathrm{L}$; platelet count $\geq 100 \times 10^{9} / \mathrm{L}$, and normal liver and kidney function. Exclusion criteria included: presence of multiple tumors; histologically proven adenocarcinoma or other types of cancer; distant metastases; comprehensive evaluation by two chief physicians of patients with signs of esophageal perforation before treatment; previous esophageal surgery; incomplete medical records or imaging data. The clinical staging of esophageal cancer was based on the guidelines of the International Union Against Cancer (UICC), 8th Edition.

\section{Treatment}

For all patients, the RT technique used was SIB radiation therapy. Gross tumor volume (GTV), clinical target volume (CTV), planning target volume (PTV), planning gross tumor volume (PGTV), spinal cord, lung, and heart were delineated. GTV included $G_{T} V_{T}$ and $G_{T V}$. $G_{T V}$ was a gross tumor of the esophagus, and $\mathrm{GTV}_{\mathrm{N}}$ was a positive lymph node. CTV was enlarged $3 \mathrm{~cm}$ in the upper and lower direction of $\mathrm{GTV}_{\mathrm{T}}$ and $\mathrm{GTV}_{\mathrm{N}}, 0.5 \mathrm{~cm}$ in the left and right direction of $\mathrm{GTV}_{\mathrm{T}}$, and enlarged lymph node involved area, which was modified appropriately according to the anatomical structure. PTV is the outward expansion of $0.5 \mathrm{~cm}$ around CTV. PGTV was created at a $0.5 \mathrm{~cm}$ radial margin from GTV, 95\% PTV median prescription dose: 5400 cGy 30 fraction (180 cGy per fraction); PGTV median dose: 6000 cGy 30 fraction (200 cGy per fraction). The RT dose for patients with cervical esophageal cancer was PGTV: 6000-6600 cGy, and the median dose was $6400 \mathrm{cGY}$.

\section{Clinical Evaluation and Follow-Up}

According to the toxicity criteria of the Radiation Therapy Oncology Group (RTOG), tumor regression was evaluated 1 month after completion of treatment. All evaluated patients who achieved a clinical partial response (PR) or clinical complete response (CR) were included in the objective response rate (ORR). All patients were followed up every 3 months for the first 2 years, then every 6 months for 3-5 years. Review methods included chest and abdominal computed tomography (CT), contrast esophagography, abdominal ultrasound, bone scans, positron emission tomography scans, and puncture biopsy (if feasible).

\section{Statistical Analysis}

We defined OS as the time between diagnosis and death or the time of analysis. PFS was defined as the time from diagnosis to the first event of local failure, progression or metastatic recurrence.

IBM SPSS 26.0 software was used for statistical analysis. The chi-squared test was used to compare differences between groups. The Kaplan-Meier method was used to compute OS and PFS, and differences were compared using Log rank testing. Factors with potential significance $(\mathrm{P}<0.15$ on univariate analysis) were included in a multivariate analysis. A value of $\mathrm{P}<0.05$ was considered to be statistically significant. 


\section{Results}

\section{Demographics}

In total, 124 patients received double-agent chemoradiotherapy (CRT); taxane or fluorouracil combined with platinum were used as simultaneous medications during RT (124/315; $39.4 \%$ ). Oral S-1 or capecitabine alone was mainly used for the single-agent CRT group $(117 / 315 ; 37.1 \%) .23 .5 \%$ of patients received RT alone. 175 patients did not receive adjuvant chemotherapy, while the remaining patients received several cycles of adjuvant chemotherapy (140/315; 44.4\%).

A total of 315 patients were included in the analysis. Patient characteristics are listed in Table 1. In total, 187 patients $(59.4 \%)$ were aged $>70$ years, with a median age of 73 years. $59.4 \%$ of patients were designated as clinical

Table I Patient Characteristics

\begin{tabular}{|l|l|c|}
\hline Variable & & N (\%) \\
\hline Sex & Male & $219(69.5)$ \\
& Female & $96(30.5)$ \\
\hline Age (years) & $\leq 70$ & $128(40.6)$ \\
& $>70$ & $187(59.4)$ \\
\hline Tumor location & Cervical & $28(8.9)$ \\
& Upper & $92(29.2)$ \\
& Med & $128(40.6)$ \\
& Distal & $67(21.3)$ \\
\hline Tumor length (cm) & $\geq 6.5$ & $176(55.9)$ \\
& $<6.5$ & $139(44.1)$ \\
\hline T-stage & TI-2 & $71(25.2)$ \\
& T3 & $122(38.7)$ \\
& T4 & $122(38.7)$ \\
\hline N-stage & No & $186(59.0)$ \\
& NI & $88(27.9)$ \\
& N2 & $31(9.8)$ \\
& N3 & $10(3.2)$ \\
\hline RT technique & I-II & $128(40.6)$ \\
& III-IVa & $197(59.4)$ \\
\hline Synchical stage & SIB & $315(100)$ \\
\hline & IFI & $196(62.2)$ \\
& ENI & $119(37.8)$ \\
\hline & dCCRT & $124(39.4 .4)$ \\
& SCCRT & $117(37.1)$ \\
& RT alone & $(23.5)$ \\
\hline
\end{tabular}

Abbreviations: CTV, clinical target volume; dCCRT, double chemotherapy concurrent chemoradiotherapy; RT, radiotherapy; sCCRT, single chemotherapy concurrent chemoradiotherapy; SIB, simulated integrated boost. stage III-IVa. The primary tumor location (as determined by the upper edge of the tumor) was mainly in the middle thoracic region, accounting for $40.6 \%$ of the total. 241 patients received concurrent chemoradiotherapy, and the remaining patients received no chemotherapy.

\section{Efficacy}

The final day of follow-up was on May 30, 2021. The median follow-up time was 42.1 months (interquartile range, 34.4-53.0 months), and the follow-up rate was 96.8\%. 2-year, 3-year OS and PFS of the entire group were $49.5 \%, 40.2 \%$ and $40.3 \%, 34.0 \%$, respectively. The median OS was 23.5 months in this study. In the RT, sCCRT and dCCRT groups, the median survival times were 16.0, 26.2, and 30.2 months, respectively. A total of 118 patients $(37.5 \%)$ were alive at the end of the study period. The ORR (CR+ PR) was $87.6 \%$.

\section{Univariate and Multivariate Analysis}

Results of univariate and multivariate analyses for all relevant clinical features are shown in Tables 2 and 3. The results show that T-stage, $\mathrm{N}$-stage, clinical stage, early response, and concurrent chemotherapy were independent factors affecting OS (Figures 1-3) and PFS. Both single chemotherapy agent-CCRT (sCCRT) and double chemotherapy agent-CCRT (dCCRT) showed significant survival benefits compared with RT alone (Figure 4), but there was no significant difference between the different chemotherapy regimens.

\section{Adverse Reactions}

Regarding grade 2-4 acute adverse reactions, there were 78 cases of radiation esophagitis (24.8\%), 101 cases of leukopenia (32.1\%), 66 cases of nausea and vomiting (21.0\%), 14 cases of radiation pneumonia (4.4\%), nine cases of thrombocytopenia (2.9\%), and nine cases of hemoglobin decrease $(2.9 \%)$. All acute adverse reactions were resolved after symptomatic supportive treatment. Adverse reactions increased significantly with increasing chemotherapy intensity during RT (please see Table 4 for a detailed adverse reaction).

\section{Failure Patterns}

In total, 210 patients failed treatment, including $170(81.0 \%)$ who experienced local failure, $18(8.6 \%)$ who experienced local uncontrolled disease, and 48 (22.9\%) who experienced distant metastasis. Twenty (9.5\%) patients experienced both local failure and distant metastasis. Sites of distant 
Table 2 Univariate Analysis of Patient and Treatment Characteristics in Esophageal Cancer

\begin{tabular}{|c|c|c|c|c|c|}
\hline \multirow[t]{2}{*}{ Characteristics } & \multirow[t]{2}{*}{$\mathbf{N}$} & \multicolumn{3}{|c|}{ OS (\%) } & \multirow[t]{2}{*}{ P-value } \\
\hline & & I-Year & 2-Year & 3-Year & \\
\hline Age & & & & & 0.602 \\
\hline$>70$ & 187 & 75.9 & 49.1 & 42.9 & \\
\hline$\leq 70$ & 128 & 74.2 & 50.0 & 36.5 & \\
\hline Sex & & & & & 0.191 \\
\hline Male & 219 & 74.9 & 46.0 & 37.5 & \\
\hline Female & 96 & 76.0 & 57.3 & 46.4 & \\
\hline Tumor location & & & & & 0.141 \\
\hline Cervical-upper & 123 & 75.2 & 55.4 & 44.5 & \\
\hline Middle-lower & 192 & 75.3 & 45.8 & 37.7 & \\
\hline CCRT & & & & & 0.003 \\
\hline dCCRT & 124 & 77.4 & 57.3 & 45.6 & \\
\hline sCCRT & 117 & 81.2 & 51.2 & 41.9 & \\
\hline RT alone & 74 & 62.2 & 33.8 & 29.5 & \\
\hline T-stage & & & & & $<0.001$ \\
\hline $\mathrm{I}-2$ & 71 & 87.3 & 74.6 & 58.9 & \\
\hline 3 & 122 & 77.0 & 46.6 & 38.3 & \\
\hline 4 & 122 & 66.4 & 37.7 & 31.6 & \\
\hline $\mathrm{N}$-stage & & & & & $<0.001$ \\
\hline No & 186 & 79.6 & 54.8 & 44.6 & \\
\hline NI & 88 & 75.0 & 52.3 & 41.7 & \\
\hline N2 & 31 & 61.3 & 25.8 & 22.6 & \\
\hline N3 & 10 & 40.0 & 0.0 & 0.0 & \\
\hline Clinical stage & & & & & $<0.001$ \\
\hline I-II & 128 & 85.2 & 67.1 & 53.3 & \\
\hline III-IVa & 187 & 68.4 & 37.4 & 31.4 & \\
\hline Range of CTV & & & & & 0.208 \\
\hline IFI & 196 & 74.5 & 47.4 & 37.6 & \\
\hline ENI & 119 & 76.5 & 52.9 & 44.5 & \\
\hline $\begin{array}{l}\text { Adjuvant } \\
\text { chemotherapy }\end{array}$ & & & & & 0.565 \\
\hline No & 175 & 75.4 & 48.5 & 38.5 & \\
\hline Yes & 140 & 75.0 & 50.7 & 42.3 & \\
\hline $\begin{array}{l}\text { Tumor early } \\
\text { response }\end{array}$ & & & & & $<0.001$ \\
\hline CR/PR & 276 & 84.4 & 56.5 & 45.9 & \\
\hline SD/PD & 39 & 10.3 & 0.0 & 0.0 & \\
\hline
\end{tabular}

Abbreviations: CCRT, chemotherapy concurrent chemoradiotherapy; CR, complete response; CTV, clinical target volume; dCCRT, double chemotherapy concurrent chemoradiotherapy; ENI, elective nodal irradiation; IFI, involved-field irradiation; OS, overall survival; PD, progressive disease; PR, partial response; RT, radiotherapy; sCCRT, single chemotherapy concurrent chemoradiotherapy; SD, stable disease.

metastasis included the lung $(17 / 48 ; 35.4 \%)$, liver $(12 / 48$; $25.0 \%)$, bone $(15 / 48 ; 31.3 \%)$, and lymph node $(6 / 48$;
Table 3 Multivariate Analysis of Prognostic Factors on Treatment Outcomes in Esophageal Cancer

\begin{tabular}{|l|c|c|}
\hline Factor & P-value & HR (95\% CI) \\
\hline $\begin{array}{l}\text { Location (cervical-upper vs } \\
\text { middle-lower) }\end{array}$ & 0.535 & $\mathrm{I} .100(0.813-\mathrm{I} .487)$ \\
\hline T-stage (I-2 vs 3-4) & 0.049 & $\mathrm{I} .573(\mathrm{I} .002-2.468)$ \\
\hline N-stage (0-I vs 2-3) & 0.024 & $\mathrm{I} .594(\mathrm{I} .064-2.387)$ \\
\hline AJCC stage (I-II vs III-IVa) & $0.04 \mathrm{I}$ & $\mathrm{I} .448(\mathrm{I} .014-2.067)$ \\
\hline $\begin{array}{l}\text { Concurrent chemotherapy (Yes } \\
\text { vs No) }\end{array}$ & $<0.00 \mathrm{I}$ & $\mathrm{I} .792(\mathrm{I} .298-2.472)$ \\
\hline RT effect (CR/PR vs SD/PD) & $<0.00 \mathrm{I}$ & $0.098(0.064-0.149)$ \\
\hline
\end{tabular}

Abbreviations: AJCC, American Joint Committee on Cancer; $\mathrm{Cl}$, confidence interval; CR, complete response; HR, hazard ratio; PD, progressive disease; PR, partial response; SD, stable disease.

$12.5 \%)$. The main mode of recurrence of esophageal cancer was local failure, followed by vascular metastasis.

\section{Discussion}

Analysis of failure patterns in esophageal cancer patients receiving definitive CRT revealed that local failure occurred in $50 \%$ of patients. ${ }^{6}$ Of these patients, approximately $90 \%$ of progression occurred within the GTV volume. It has been speculated that increasing RT dose may lead to more effective local control of esophageal cancer. The development of SIB-IMRT RT technology has made it possible to increase the local radiation dose to solid tumors. According to the results of previous research, SIB-IMRT significantly reduced the failure rate in patients with positive lymph nodes compared with IMRT ( $13 \%$ vs $56 \%$; $\mathrm{P}=0.04){ }^{7}$

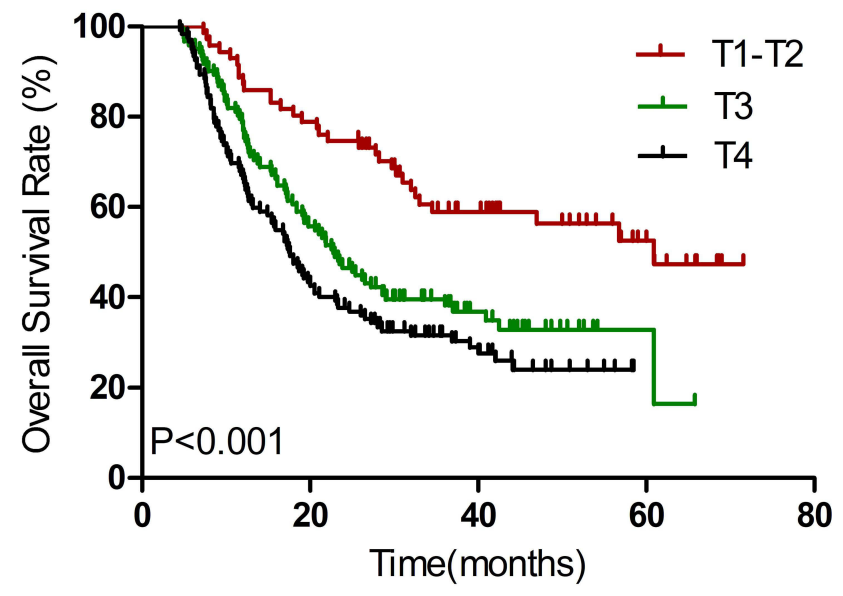

Figure I Kaplan-Meier estimates of overall survival for TI-T2 vs T3 vs T4. 


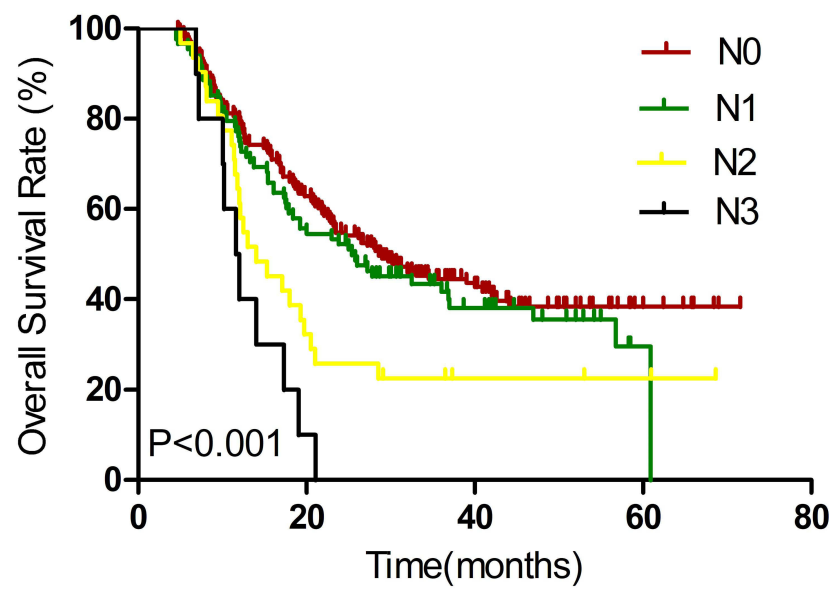

Figure 2 Kaplan-Meier estimates of overall survival for N0 vs NI vs N2 vs N3.

The 1-year and 3-year OS rates in our study were $75.2 \%$ and $40.2 \%$, respectively, which were substantially higher than those reported by Ristau et al $(63.9 \%$ and $37.6 \%$ respectively). ${ }^{8}$ This result is in line with the findings of $\mathrm{Xu}$ et al, ${ }^{9}$ but considerably lower than the 3- and 5 -year survival rates of $53 \%$ and $44 \%$ and the median survival time of 43.2 months with IMRT reported by Lin et al. ${ }^{10} \mathrm{~A}$ possible explanation for this difference is that only $76.5 \%$ of the patients received CCRT in our study.

CCRT, as the standard of care, has been demonstrated in the RTOG 8501 and JOCG9906 studies to provide encouraging survival results and acceptable RT-related toxicity in patients with inoperable esophageal cancer. $^{11,12}$ In our study, patients who received CCRT had better survival outcomes than those treated with RT alone, with a median survival time of 30.2 months in the dCCRT group, 26.2 months in the sCCRT group, and 16 months in the RT group $(\mathrm{P}=0.003)$. Efficacy in the dCCRT

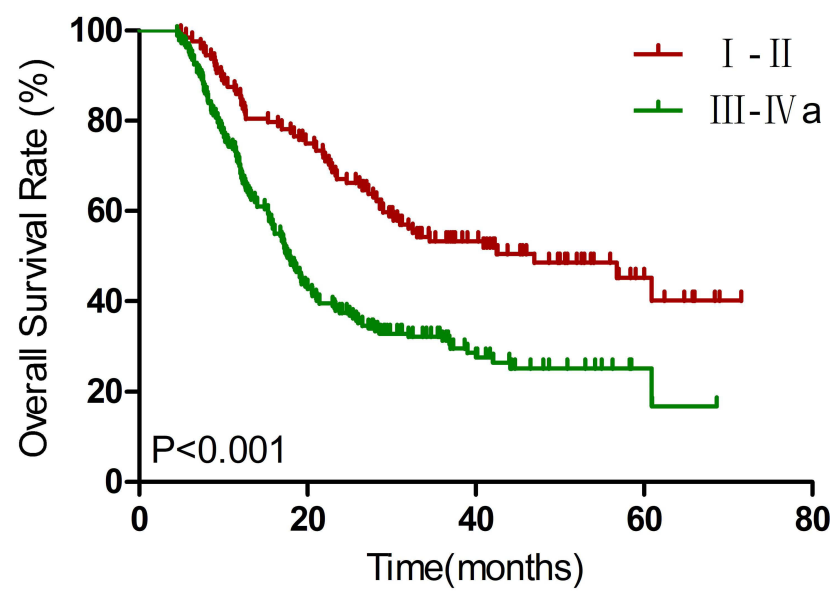

Figure 3 Kaplan-Meier estimates of overall survival for I-II stage vs III-IVa stage.

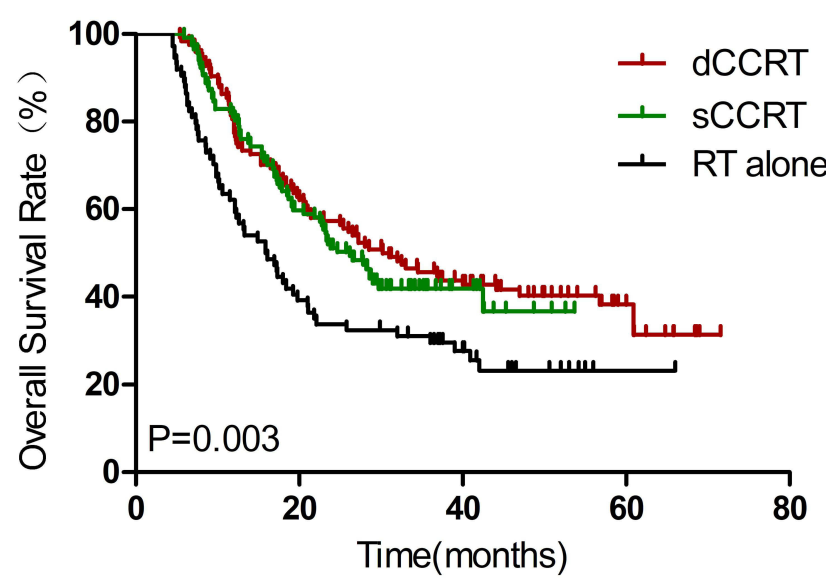

Figure 4 Kaplan-Meier estimates of overall survival for sCCRT vs dCCRT vs RT alone.

group was similar to that of the sCCRT group, although more grade 2-4 severe toxicities were observed in the dCCRT group, including myelosuppression, leucopenia, radioactive esophagitis, and gastrointestinal reaction. Differences in intensity of chemotherapy during RT did not affect the incidence of radiation pneumonia, which was essentially consistent with the results of $\mathrm{Li}$ et al. ${ }^{13}$ The results of Phase II clinical trial reported by Zhao et al ${ }^{14}$ showed that the median disease-free survival times were 20 and 21 months for the single-agent and dual agent groups, respectively, with no significant differences. However, the incidence of myelosuppression and vomiting was significantly lower in the single-agent group. Severe (grade $>2$ ) radiation pneumonitis was very rare $(<4.5 \%)$ and the rate of grade 2-4 esophagitis was less than $25 \%$ in our study, which might be due to the advanced SIB-IMRT technique and optimal treatment plan utilized.

In our research, Univariate analysis and multivariate Cox regression analysis have shown that short-term efficacy, T-stage, ${ }^{15,16} \mathrm{~N}$-stage, ${ }^{17}$ and clinical stage ${ }^{18}$ were independent prognostic factors in esophageal cancer. In general, the higher the N-stage, the more extensive the tumor invasion, leading to a poorer prognosis. Early response is an essential factor affecting prognosis, and the prognosis of patients with $\mathrm{CR} / \mathrm{PR}$ is significantly better than that of patients with stable disease (SD) or progressive disease (PD). Because early efficacy reflects the sensitivity of the tumor to RT, patients with esophageal cancer who are more sensitive to RT have a higher ORR, resulting in a better prognosis, as demonstrated in several studies. ${ }^{19-21}$ At present, the role of adjuvant chemotherapy is not clear. Postoperative adjuvant chemotherapy is 
Table 4 Treatment-Related Toxicities

\begin{tabular}{|c|c|c|c|c|c|c|c|}
\hline \multirow[t]{2}{*}{ Toxicities, N (\%) } & \multicolumn{2}{|c|}{ RT Alone } & \multicolumn{2}{|c|}{ Single-Agent CRT } & \multicolumn{2}{|c|}{ Double-Agent CRT } & \multirow[t]{2}{*}{ P-value } \\
\hline & Grade 0-I & $\geq$ Grade 2 & Grade 0-I & $\geq$ Grade 2 & Grade 0-I & $\geq$ Grade 2 & \\
\hline Myelosuppression & $63(85.1)$ & II (I4.9) & $90(76.9)$ & $27(23.1)$ & $53(42.7)$ & 7I (57.3) & $<0.001$ \\
\hline Leukopenia & $65(87.8)$ & $9(12.2)$ & $92(78.6)$ & $25(21.4)$ & $57(46.0)$ & $67(54.0)$ & $<0.001$ \\
\hline Neutropenia & $65(87.8)$ & $9(12.2)$ & 91 (77.8) & $26(22.2)$ & $56(45.2)$ & $68(54.8)$ & $<0.001$ \\
\hline Thrombocytopenia & $73(98.6)$ & I (I.4) & II 5 (98.3) & $2(1.7)$ & II 8 (95.2) & $6(4.8)$ & 0.314 \\
\hline Anemia & $72(97.3)$ & $2(2.7)$ & $115(98.3)$ & $2(1.7)$ & $119(96.0)$ & $5(4.0)$ & 0.633 \\
\hline Esophagitis & $62(83.8)$ & $12(16.2)$ & $93(79.5)$ & $24(20.5)$ & $82(66.1)$ & $42(33.9)$ & 0.008 \\
\hline Radiation pneumonitis & 71 (95.9) & $3(4.1)$ & $113(96.6)$ & $4(3.4)$ & $117(94.4)$ & $7(5.6)$ & 0.737 \\
\hline Gastrointestinal reaction & $70(94.6)$ & $4(5.4)$ & $106(88.9)$ & II (9.4) & $73(58.9)$ & $5 I(4 I . I)$ & $<0.001$ \\
\hline
\end{tabular}

Abbreviations: CRT, chemoradiotherapy; RT, radiotherapy.

recommended for esophageal cancer and adenocarcinoma at the esophagogastric junction. ${ }^{22}$ For squamous cell carcinoma, some studies have shown that adjuvant chemotherapy can prolong PFS but has no significant effect on OS. ${ }^{23,24}$ In our research of definite RT, adjuvant chemotherapy did not show a significant survival benefit. Adjuvant chemotherapy is considered to eliminate circulating tumor cells in the blood to prevent distant metastasis of the tumor. ${ }^{25}$ However, recurrence of esophageal cancer typically occur in situ, and chemotherapy therefore needs to reach the tumor tissue through blood circulation. As the esophageal blood supply after RT is reduced, the survival benefit is diminished.

There are certain limitations in our study. First, a potential bias exists due to the retrospective design, while the follow-up time may not have been long enough to reach definitive conclusions. In addition, the chemotherapy regimen and number of chemotherapy cycles during RT were not uniform between patients.

\section{Conclusion}

We have established SIB-IMRT as a relatively effective treatment for esophageal cancer, with good long-term efficacy and controllable adverse reactions. dCCRT is equally effective but more toxic than sCCRT. T-stage, N-stage, clinical stage, concurrent chemotherapy, and early response were independent prognostic factors affecting survival.

\section{Ethics Approval and Informed Consent}

The study was conducted in accordance with the Helsinki Declaration and was approved by the ethics committee of The First Affiliated Hospital of Bengbu Medical College
(2021KY032). We guarantee that all patient data will be kept confidential.

\section{Acknowledgments}

This work was supported by Key Project of Translational Medicine in Bengbu Medical College (BYTM2019027).

\section{Disclosure}

The authors declare no conflicts of interest.

\section{References}

1. Sung H, Ferlay J, Siegel RL, et al. Global cancer statistics 2020: GLOBOCAN estimates of incidence and mortality worldwide for 36 cancers in 185 countries. CA Cancer J Clin. 2021;71:209-249. doi: $10.3322 /$ caac. 21660

2. Morita M, Yoshida R, Ikeda K, et al. Advances in esophageal cancer surgery in Japan: an analysis of 1000 consecutive patients treated at a single institute. Surgery. 2008;143(4):499-508. doi:10.1016/j. surg.2007.12.007

3. Chiu PW, Chan AC, Leung SF, et al. Multicenter prospective randomized trial comparing standard esophagectomy with chemoradiotherapy for treatment of squamous esophageal cancer: early results from the Chinese University Research Group for Esophageal Cancer (CURE). J Gastrointest Surg. 2005;9(6):794-802. doi:10.1016/j. gassur.2005.05.005

4. Zhang WZ, Chen JZ, Li DR, et al. Simultaneous modulated accelerated radiation therapy for esophageal cancer: a feasibility study. World J Gastroenterol. 2014;20:13973-13980. doi:10.3748/wjg.v20. i38.13973

5. Welsh J, Palmer MB, Ajani JA, et al. Esophageal cancer dose escalation using a simultaneous integrated boost technique. Int $J$ Radiat Oncol Biol Phys. 2012;82(1):468-474. doi:10.1016/j. ijrobp.2010.10.023

6. Welsh J, Settle SH, Amini A, et al. Failure patterns in patients with esophageal cancer treated with definitive chemoradiation. Cancer. 2012;118(10):2632-2640. doi:10.1002/cncr.26586

7. Welsh JW, Seyedin SN, Allen PK, et al. Local control and toxicity of a simultaneous integrated boost for dose escalation in locally advanced esophageal cancer: interim results from a prospective phase I/II trial. $J$ Thorac Oncol. 2017;12(2):375-382. doi:10.1016/j. jtho.2016.10.013 
8. Ristau J, Thiel M, Katayama S, et al. Simultaneous integrated boost concepts in definitive radiation therapy for esophageal cancer: outcomes and toxicity. Radiat Oncol. 2021;16(1):23. doi:10.1186/ s13014-021-01749-x

9. Xu Y, Wang Z, Liu G, et al. The efficacy and safety of simultaneous integrated boost intensity-modulated radiation therapy for esophageal squamous cell carcinoma in Chinese population: a single institution experience. J Cancer Res Ther. 2016;12(5):82-88. doi:10.4103/09731482.191640

10. Lin S, Wang L, Myles B, et al. Propensity score-based comparison of long-term outcomes with 3-dimensional conformal radiotherapy vs intensity-modulated radiotherapy for esophageal cancer. Int J Radiat Oncol Biol Phys. 2012;84(5):1078-1085. doi:10.1016/j. ijrobp.2012.02.015

11. Cooper JS, Guo MD, Herskovic A, et al. Chemoradiotherapy of locally advanced esophageal cancer: long-term follow-up of a prospective randomized trial (RTOG 85-01). Radiation Therapy Oncology Group. JAMA. 1999;281(17):1623-1627. doi:10.1001/ jama.281.17.1623

12. Kato K, Muro K, Minashi K, et al. Phase II study of chemoradiotherapy with 5-fluorouracil and cisplatin for stage II-III esophageal squamous cell carcinoma: JCOG trial (JCOG 9906). Int J Radiat Oncol Biol Phys. 2011;81:684-690. doi:10.1016/j.ijrobp.2010.06.033

13. Li J, Gong Y, Diao P, et al. Comparison of the clinical efficacy between single-agent and dual-agent concurrent chemoradiotherapy in the treatment of unresectable esophageal squamous cell carcinoma: a multicenter retrospective analysis. Radiat Oncol. 2018;13(1):12. doi:10.1186/s13014-018-0958-5

14. Zhao Z, Wen Y, Liao D, et al. Single-agent versus double-agent chemotherapy in concurrent chemoradiotherapy for esophageal squamous cell carcinoma: prospective, randomized, multicenter phase II clinical trial. Oncologist. 2020;25(12):e1900-e1908. doi:10.1634/ theoncologist.2020-0808

15. Jiang N, Ge XL, Zhang ZY, et al. Prognostic factors for patients with esophageal cancer receiving definitive radiotherapy alone: a retrospective analysis. Cancer Manag Res. 2021;13:3229-3234. doi:10.2147/CMAR.S300672

16. Zhao L, Zhou Y, Pan H, et al. Radiotherapy alone or concurrent chemoradiation for esophageal squamous cell carcinoma in elderly patients. J Cancer. 2017;8(16):3242-3250. doi:10.7150/jca.20835
17. Yin H, Li D, Zhu C, et al. Factors relevant to the prognosis of patients with esophageal cancer who received intensity-modulated radiotherapy. Thorac Cancer. 2018;9(10):1215-1219. doi:10.1111/ 1759-7714.12800

18. Du XX, Yu R, Wang ZF, et al. Outcomes and prognostic factors for patients with cervical esophageal cancer undergoing definitive radiotherapy or chemoradiotherapy. Bosn J Basic Med Sci. 2019;19:186-194.

19. Huang C, Zhu Y, Li Q, et al. Feasibility and efficiency of concurrent chemoradiotherapy with a single agent or double agents vs radiotherapy alone for elderly patients with esophageal squamous cell carcinoma: experience of two centers. Cancer Med. 2019;8 (1):28-39. doi:10.1002/cam4.1788

20. Yeom JG, Kim J-H, Kim JW, et al. Prognostic significance of interim response evaluation during definitive chemoradiotherapy for locally advanced esophageal squamous cell carcinoma. Cancers. 2021;13 (6):1255. doi:10.3390/cancers 13061255

21. Chen M, Liu P, Chen Y, et al. Primary tumor regression patterns in esophageal squamous cell cancer treated with definitive chemoradiotherapy and implications for surveillance schemes. Cancer Manag Res. 2019;11:3361-3369. doi:10.2147/CMAR.S198524

22. Cunningham D, Allum WH, Stenning SP, et al. Perioperative chemotherapy versus surgery alone for resectable gastroesophageal cancer. $N$ Engl J Med. 2006;355(1):11-20. doi:10.1056/ NEJMoa055531

23. Ando N, Iizuka T, Ide H, et al. Surgery plus chemotherapy compared with surgery alone for localized squamous cell carcinoma of the thoracic esophagus: a Japan Clinical Oncology Group StudyJCOG9204. J Clin Oncol. 2003;21:4592-4596. doi:10.1200/ JCO.2003.12.095

24. Zhang L, Li W, Lyu X, et al. Adjuvant chemotherapy with paclitaxel and cisplatin in lymph node-positive thoracic esophageal squamous cell carcinoma. Chin J Cancer Res. 2017;29(2):149-155. doi:10.21147/j.issn.1000-9604.2017.02.08

25. Xia X, Liu Z, Qin Q, et al. Long-term survival in nonsurgical esophageal cancer patients who received consolidation chemotherapy compared with patients who received concurrent chemoradiotherapy alone: a systematic review and meta-analysis. systematic review. Front Oncol. 2021;10:604657. doi:10.3389/fonc.2020.604657

\section{Publish your work in this journal}

Cancer Management and Research is an international, peer-reviewed open access journal focusing on cancer research and the optimal use of preventative and integrated treatment interventions to achieve improved outcomes, enhanced survival and quality of life for the cancer patient.
The manuscript management system is completely online and includes a very quick and fair peer-review system, which is all easy to use. Visit http://www.dovepress.com/testimonials.php to read real quotes from published authors. 